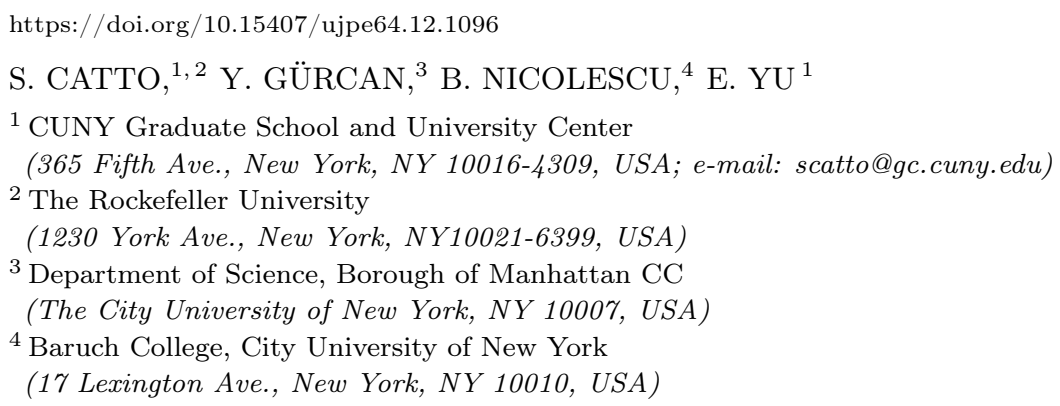

\title{
EFFECTIVE HADRONIC SUPERSYMMETRY FROM QUANTUM CHROMODYNAMICS ${ }^{1}$
}

\begin{abstract}
A quark model with potentials derived from QCD that include antiquark-diquark model for excited hadrons leads to mass formulae in very good agreement with experiments. The approximate symmetries and supersymmetries of the hadronic spectrum are exploited including a symmetry breaking mechanism.

Ke ywords: supersymmetry, quark models, skyrmions.
\end{abstract}

\section{Introduction}

In 1966-1968, Miyazawa extended, in a series of papers, the SU(6) group [1] to the supergroup SU(6/21). Elements of this supergroup transform constituent quarks and diquarks to one another. In particular, in $[2,3]$, he found: $(a)$ the general definition of $\mathrm{SU}(m / n)$ superalgebras expressing the symmetry between $m$ bosons and $n$ fermions, with Grassmann-valued parameters, $(b)$ the derivation of a super-Jacobi identity, and $(c)$ the relation of the baryon mass splitting to the meson mass splitting through a new mass formula. That work contained the first classification of superalgebras (later rediscovered by mathematicians in the seventies). Because of the field theoretic prejudice against SU(6), Miyazawa's work was generally ignored. Supersymmetry was rediscovered in the seventies within the context of the dual resonance models by Ramond [4], Neveu and Schwarz [5], with the important later contributions of Gliozzi, Scherk, and Olive [6, 7]. Golfand and Likthman [8] and independently Volkov and Akulov [9] proposed the extension of the Poincaré group to the superPoincaré group. Examples of supersymmetric field theories were given and the general method based on the super-Poincaré group was discovered by Wess and Zumino $[10,11]$. QCD can be made supersymmetric

(c) S. CATTO, Y. GÜRCAN, B. NICOLESCU, E. YU, 2019 1096 with the addition of gluinos and squarks which remain to be discovered. It gives no help for throwing light on the supersymmetric nature of the slope of the Regge trajectories. The super-Poincaré group contains transformations between fields associated with different spins $0, \frac{1}{2}$ and 1 . The Coleman-Mandula theorem was amended in 1975 by Haag, Lopuszanski, and Sohnius who showed that the super-Poincaré group $\times G_{\text {int }}$ is the maximal symmetry of the $S$-matrix. Unfortunately, the SU(6) symmetry was still forbidden.

\section{SU(6) and Hadronic Supersymmetry}

To interpret the symmetries of the QCD spectrum under a new light, we made the following observations: in the ultraviolet, the running coupling constant tends to zero, and quarks behave like free particles. Thus, an approximate scaling symmetry exists in conformity with the parton model, allowing spin to be conserved seperately from orbital angular momentum. Spin behaves as an internal quantum number; this makes the $\mathrm{SU}(6)$ symmetry possible, since the quarks are almost free Dirac particles. The single vector-gluon exchange breaks this symmetry; thus, as shown by Glashow, Georgi, and deRujula [12], the

1 This work is based on the results presented at the XI BolyaiGauss-Lobachevskii (BGL-2019) Conference: Non-Euclidean, Noncommutative Geometry and Quantum Physics.

ISSN 2071-0194. Ukr. J. Phys. 2019. Vol. 64, No. 12 
mass degeneracy of hadrons of different spins is lifted by a hyperfine-interaction term.

The main point of the idea can be understood in the infrared, where the confinement sets in. The quark-antiquark potential becomes proportional to the distance. Careful studies of quarkonium spectra and lattice-gauge calculations show that, at a large separation, the quark forces become spin-independent. QCD is also flavor-independent. Therefore, we will seach for approximate spin- and flavorindependent quark binding forces. These forces are completely consistent with the $\mathrm{SU}(6)$ symmetry. This symmetry is not exact, but it is a good starting point before the spin and flavor effects are included.

There is a good phenomenological evidence that, in a rotationally excited baryon, a quark-diquark $((q-D)$ structure is favored [13] over a three quark ( $q q q)$ structure [13-15]. Regge trajectories for mesons and baryons are closely parallel; both have a slope of about $0.9 \mathrm{GeV}^{-2}$ (a proposal to find this result in QCD was put forth by Johnson and Thorn $[16,17])$. At large spins, two of the quarks form a diquark at one end of the string, the remaining quark being at the other.

If the quarks are light, the underlying quarkdiquark symmetry leads to the Miyazawa symmetry between mesons and baryons. Thus, we may attempt to study hadrons with the weakly broken supergroup $\mathrm{SU}(6 / 21)$, even though the fundamental theory is not supersymmetric. For quarks, the generators of the Poincaré group and those of the color group $\mathrm{SU}(3)^{c}$ commute. It is only the effective Hamiltonian which exhibits an approximate supersymmetry among the bound states $q \bar{q}$ and $q D(D=q q)$.

A lot of remarkable papers were put out by Brodsky and collaborators in recent times [14-20] on the diquark physics using superconformal and supersymmetric algebras through the embedding of superconformal quantum mechanics into the $A d S$ space. They show the possibility of constructing an effective supersymmetric QCD light-front Hamiltonian for hadrons, exhibiting relations between meson, baryon, and tetraquark spectra with emphasis on the connections between light, heavy-light, and doubleheavy hadrons. Their work has immense relationship to our own work from a slightly different perspective. We refer the reader to glance at their papers and see the thorough relationship to the latest experimental searches and findings on this subject.

ISSN 2071-0194. Ukr. J. Phys. 2019. Vol. 64, No. 12

\section{Color Algebra and Octonions}

The exact unbroken color group $\mathrm{SU}(3)^{c}$ is the backbone of the strong interaction. It is worthwhile to understand its role in the diquark picture more clearly.

Two of the colored quarks in a baryon combine into an antitriplet $3 \times 3=\overline{3}+(6), 3 \times \overline{3}=1+(8)$. The $(6)$ partner of the diquark and the (8) partner of the nucleon do not exist. In the hadron dynamics, the only color combinations to consider are $3 \times 3 \rightarrow \overline{3}$ and $\overline{3} \times 3 \rightarrow 1$. These relations yield the existence of split octonion units through a representation of the Grassmann algebra $\left\{u_{i}, u_{j}\right\}=0, i=1,2,3$. The operators $u_{i}$, unlike ordinary fermion operators, are not associative. We also have $\frac{1}{2}\left[u_{i}, u_{j}\right]=\epsilon_{i j k} u_{k}^{*}$. The Jacobi identity do not hold, since $\left[u_{i},\left[u_{j}, u_{k}\right]\right]=-\sigma i e_{7} \neq 0$, where $e_{7}$ anticommutes with $u_{i}$ and $u_{i}^{*}$.

The algebra of split octonions is crucial for the suppression of unwanted states and is fully discussed in our papers [25-27] in detail. The automorphism group of the algebra is just $\mathrm{SU}(3)^{c}$. It is a subgroup of $G_{2}$, the automorphism group of the octonion (Cayley) algebra. Gürsey and collaborators discovered a link between color and octonions, earlier in connection with a grand-unified theory of leptons and quarks with a natural color embedding (the non-colored part has units $u_{0}=\frac{1}{2}\left(1+i e_{7}\right)$ and $u_{0}^{*}=\frac{1}{2}\left(1-i e_{7}\right)$ associated with the lepton number). We ask the interested reader to glance through our papers.

\section{Internal SUSY and Classification Schemes}

Using the internal supersymmetry, it is possible to construct a combined classification scheme for mesons and baryons with Miyazawa's $\mathrm{SU}(6 / 21)$ in a modern context. We have already shown $[14,15]$ : (1) the parallelism and the splitting of Regge trajectories for mesons and baryons can be calculated at high angular momenta. (2) Hadronic mass formulas can be derived, which agree remarkably well with experiments [15]. This scheme naturally leads to the existence of $D \bar{D}(D=q q, \bar{D}=\bar{q} \bar{q})$ bound states. It is now confirmed experimentally [28] that $a_{0}(980)$ and $f_{0}(975)$ mesons are $D \bar{D}$ states. (3) There is also a deep connection between the hadronic $(\mathrm{SU}(6 / 21))$ scheme we have developed and the mass formulae derived from spectrum-generating algebras which also assume an effective supersymmetry $[29,30]$. The fact that both supersymmetries (in hadrons and nuclei) are effective 
"dynamical" supersymmetries leads to many novel experimental observations. (For recent reviews, we refer to Iachello's article in [31] and an extensive review in [28].) (4) Split octonionic algebras were used to understand the suppression of unwanted states. These algebras are embedded in a new larger algebra that puts quarks, diquarks, and exotics in the same supermultiplet as hadrons. The result is to naturally suppress quark configurations that are symmetric in color and space and antisymmetric in the remaining flavor, spin, and position variables.

\section{Semirelativistic Hamiltonian}

We now present an effective Hamiltonian obtained from a two-body Schrödinger-Dirac approximation to the quark-QCD system after the elimination of the gluon degrees of freedom.

Ignoring the center-of-mass motion, we can write a semirelativistic wave equation for the wave function for a two-body system (either $q q$, or $q D) \psi_{12}(\boldsymbol{r})$ of the bilocal object with energy eigenvalues $\Omega_{12}$, namely,

$\left(\Omega_{12}-V_{c}\right) \psi_{12}=\sum_{i=1}^{2}\left\{\left[\left(m_{i}+\frac{1}{2} V_{s}\right)^{2}-\nabla^{2}\right]^{1 / 2}\right\} \psi_{12}$.

The scalar and vector potentials are given by

$V_{s}=b r, \quad V_{c}=-\frac{4}{3} \frac{\alpha_{s}}{r}+\kappa_{12} \frac{\boldsymbol{s}_{1} \boldsymbol{s}_{2}}{m_{1} m_{2}}$,

where $\frac{4}{3}$ is the color factor, $\alpha_{s}$ is the strong coupling constant at the energy $\Omega_{12}$, and the spin-dependent part of the vector potential is the hyperfine structure correction due to the gluon exchange with $\kappa_{12}=$ $=\left|\psi_{12}(0)\right|^{2}$. We see that, at large $r$, by neglecting the mass difference $\left(m_{2}-m_{1}\right)$, we find the same equation for both $(q-\bar{q})$ and $q-D$ systems, except for the presence of the hyperfine term that breaks the symmetry between $\bar{q}$ and $D$. To this approximation, we can transform the second constituent $\bar{q}$ into $D$ and vice versa without changing the energy eigenvalue $\Omega$. This means that the system admits the approximate $S U(6 / 21)$ supersymmetry transformation

$\delta \bar{q}_{\alpha}^{i}=\bar{b}_{\alpha \beta \gamma}\left(D^{i}\right)^{\beta \gamma}$,

$\delta\left(D^{i}\right)^{\beta \gamma}=b^{\alpha \beta \gamma} \bar{q}_{\alpha}^{i}$

in addition to the $\mathrm{SU}(6)$ transformation

$\delta \bar{q}_{\alpha}^{i}=m_{\alpha}^{\beta} \bar{q}_{\beta}^{i}$,

$\delta\left(D^{i}\right)^{\beta \gamma}=n_{\rho \sigma}^{\beta \gamma}\left(D^{i}\right)^{\rho \sigma}$.
The breaking of both $S U(6)$ and $S U(6 / 21)$ is due to the hyperfine term, while the supersymmetry is further broken by the quark-diquark mass difference $m_{1}-m_{2}$.

Now, considering the Hamiltonian of Eq.(1), we can write, in the center-of-mass system,

$-\nabla^{2}=\boldsymbol{p}^{2}=p_{r}^{2}+\frac{\ell(\ell+1)}{r^{2}}$,

where $\ell$ is associated with the orbital excitation of the system. For high rotational excitations, the expectation value of $r$ is large, by corresponding to a stretched string. The angular momentum $\ell$ is also large. The value of the centrifugal energy which is proportional to $\frac{\ell(\ell+1)}{r^{2}}$ has a similarly large value. Since $V_{s}$ proportional to $r$ will also have a high absolute value, the constituent masses become negligible in the high relativistic limit. On the other hand, the radial excitation term $p_{r}^{2}$ can be neglected on the leading trajectory associated with the lowest radial energy.

The ground-state energy eigenvalue $E$ of the Hamiltonian can be estimated, by using the Heisenberg uncertainty principle. This leads to the replacement of $r$ by $\Delta r$ and $p_{r}$ by

$\Delta p_{r}=\frac{1}{2}(\Delta r)^{-1} \quad(\hbar=1)$

Then $E$ as a function of $\Delta r$ is minimized for the value of $r_{0}$ of $\Delta r$. The quantity $r_{0}$ corresponds to the Bohr radius for the bound state. The confining energy associated with this Bohr radius is obtained from the linear confining potential $S(r)=b r$, so that the effective masses of the constituents become

$M_{1}=m_{1}+\frac{1}{2} S_{0}, \quad M_{2}=m_{2}+\frac{1}{2} S_{0} \quad\left(S_{0}=b r_{0}\right)$.

For a meson, $m_{1}$ and $m_{2}$ are the current quark masses while $M_{1}$ and $M_{2}$ can be interpreted as the constituent quark masses. Note that, even in the case of vanishing quark masses associated with the perfect chiral symmetry, the confinement results in nonzero constituent masses that spontaneously break the $S U(2) \times S U(2)$ symmetry of the $u$ and $d$ quarks.

Let us illustrate this method on the simplified spinfree Hamiltonian involving only the scalar potential. In the center-of-mass system, $\boldsymbol{p}^{(1)}+\boldsymbol{p}^{(2)}=0$ or $\boldsymbol{p}^{(1)}=-\boldsymbol{p}^{(2)}=\boldsymbol{p}$. The semirelativistic Hamiltonian

ISSN 2071-0194. Ukr. J. Phys. 2019. Vol. 64, No. 12 
of the system is then given by

$E_{12} \Phi=\sum_{i=1}^{2} \sqrt{\left(m_{i}+\frac{1}{2} b r\right)^{2}+\boldsymbol{p}^{2}} \Phi$.

Taking $m_{1}=m_{2}=m$ for the quark-antiquark system, we have

$E_{12} \Phi=2 \sqrt{\left(m+\frac{1}{2} b r\right)^{2}+p_{r}^{2}+\frac{\ell(\ell+1)}{r^{2}}} \Phi$,

where we have written the momentum part in spherical coordinates.

Putting

$b=\mu^{2}, \quad \rho=\mu r$

for the $q-\bar{q}$ system, we find $E_{12}$ by minimizing the function

$E_{q \bar{q}}=2 \sqrt{\left(m+\frac{1}{2} \mu \rho\right)^{2}+\frac{\mu^{2}}{\rho^{2}}\left(\ell+\frac{1}{2}\right)^{2}}$.

For $u$ and $d$ quarks, $m$ is small and can be neglected so that

$E^{2}=\mu^{2}\left[\rho^{2}+\rho^{-2}(2 \ell+1)^{2}\right]$,

which has a minimum for

$\rho^{2}=\rho_{0}^{2}=2 \ell+1$

by giving

$E_{\min }^{2}=E^{2}\left(\rho_{0}\right)=4 \mu^{2}\left(\ell+\frac{1}{2}\right)$.

Thus, we obtain a linear Regge trajectory with

$\alpha^{\prime}=\frac{1}{4} \mu^{-2}=\frac{b}{4}$.

We also have $\boldsymbol{J}=\boldsymbol{\ell}+\boldsymbol{S}$, where $\boldsymbol{S}$ arises from the quark spins. Experimentally,

$\alpha^{\prime}=0.88(\mathrm{GeV})^{-2}$

for mesons, by giving a value of $0.54 \mathrm{GeV}$ for $\mu$. A more accurate calculation (see [4]) gives

$\alpha^{\prime}=\left(2 \pi \mu^{2}\right)^{-1}, \quad \mu \sim 0.43 \mathrm{GeV}$.

The constituent quark mass can be defined in two ways

$M_{c}(\ell)=\frac{1}{2} E_{\min }=\mu \sqrt{\ell+\frac{1}{2}}$

ISSN 2071-0194. Ukr. J. Phys. 2019. Vol. 64, No. 12
$m_{c}^{\prime}(\ell)=S_{0}=\frac{1}{2} \mu \rho_{0}=\frac{\mu}{\sqrt{2}} \sqrt{\ell+\frac{1}{2}}$.

The first definition gives, for $\ell=0$,

$M_{c}=0.3 \mathrm{GeV}$ for $\mu=0.43$

for $u$ and $d$ quarks.

When the Coulomb-like terms are introduced in the simplified Hamiltonian (9) with negligible quark masses, one obtains

$E=\frac{\mu}{\rho}\left[-\bar{\alpha}+\sqrt{\rho^{4}+(2 \ell+1)^{2}}\right]$

with

$\bar{\alpha}=\frac{4}{3} \alpha_{s} \quad$ for $\quad(q \bar{q}), \quad \bar{\alpha}=\frac{2}{3} \alpha_{s} \quad$ for $\quad(q q)$.

The minimization of $E$ gives

$E_{0}=\mu u_{0}^{\frac{-1}{4}}\left(-\bar{\alpha}+\sqrt{u_{0}+(2 \ell+1)^{2}}\right)$,

where $u_{0}(\epsilon)=\rho_{0}^{4}$ so that

$u_{0}(\epsilon)=(2 \ell+1)^{2}\left(1+\frac{1}{2} \beta^{2}+\epsilon \sqrt{2} \beta \sqrt{\ell+\frac{1}{8} \beta^{2}}\right)$,

$\epsilon= \pm 1, \quad \beta=\frac{\bar{\alpha}}{(2 \ell+1)}$

The minimum $E_{0}$ is obtained for $\epsilon=-1$, by giving, to the second order in $\beta$ :

$E_{0}=\mu \sqrt{2(2 \ell+1)}\left(1-\frac{\beta}{\sqrt{2}} 3 \frac{\beta^{2}}{8}\right)$.

Linear Regge trajectories are obtained, if $\beta^{2}$ is negligible. Then, for mesons,

$E_{0}^{2}=4 \mu^{2} \ell+2 \mu^{2}(1-\sqrt{2} \bar{\alpha})$.

The quantity $\beta^{2}$ is negligible for small $\ell$, only if we take the lowest estimate for $\alpha_{s}$, giving 0.4 for $\bar{\alpha}$ in the $q \bar{q}$ case. For mesons with $u, d$ constituents, incorporating their spins through the Breit term, we obtain approximately

$m_{\rho} \simeq m_{\omega}=E_{0}+\frac{c}{4}, \quad m_{\pi}=E_{0}-\frac{3 c}{4}$,

where $c=K \frac{\Delta V}{M_{q}^{2}}$, and $M_{q}$ is the constituent quark mass. This gives

$E_{0}=\frac{\left(3 m_{\rho}+m_{\pi}\right)}{4}=0.61 \mathrm{GeV}$. 
Note that the deviation from experimental data is less than $1 \%$.

For the Regge slope being of the order of $1 \mathrm{GeV}$, the average meson mass of the same order is obtained from Eq. (26) in the linear trajectory approximation. In this approximation, $\bar{\alpha}$ should be treated like a parameter, rather than be placed by its value derived from QCD under varying assumptions. Using Eq. (17) for $\mu$, one gets a better fit to the meson masses by taking $\alpha_{s} \sim 0.2$.

Turning now to baryon masses, we must firstly estimate the diquark mass. For the $q q$ system, we have

$M_{D}=\mu\left(\sqrt{2}-\frac{2}{3} \alpha_{s}\right)$

that is slightly higher than the average meson mass

$\tilde{m}=\mu\left(\sqrt{2}-\frac{4}{3} \alpha_{s}\right)$.

Here, we note that $E$ is not very sensitive to the precise value of the QCD running coupling constant in the $\mathrm{GeV}$ range. Taking $\alpha_{s} \sim 0.3$ changes $E^{q q}$ from 0.55 to $0.54 \mathrm{GeV}$.

Note that Eq. (30) gives $m_{D}=0.55 \mathrm{GeV}$. For excited $q-\bar{q}$ and $q-D$ systems, if the rotational excitation energy is large compared with $\mu$, then both the $m_{D}$ and the Coulomb term $-\frac{4}{3} \frac{\alpha_{s}}{r}$ (same for the $q-D$ and $q-\bar{q}$ systems) can be neglected. Thus, for both the $(q-D)$ [excited baryon] and $q-\bar{q}$ [excited meson] systems, we have Eq. (14), namely,

$\left(E^{q-D}\right)^{2} \sim\left(E^{q-\bar{q}}\right)^{2} \sim 4 \mu^{2} \ell+2 \mu^{2}$.

This gives again Eq. (15), i.e.,

$\left(\alpha^{\prime}\right)_{q-D}=\left(\alpha^{\prime}\right)_{q-\bar{q}} \cong \frac{1}{4 \mu^{2}}$ or $\left(\frac{1}{2 \pi \mu^{2}}\right)$

as an explanation of the hadronic supersymmetry in the nucleon and meson Regge spectra. We also have, by extrapolating to small

$\Delta\left(M^{2}\right)^{q-D}=\Delta\left(m^{2}\right)^{q-\bar{q}}=4 \mu^{2} \Delta \ell=\frac{1}{\alpha^{\prime}} \Delta \ell$.

For $\Delta \ell=1$, we find

$m_{\Delta}^{2}-m_{N}^{2}=m_{\rho}^{2}-m_{\pi}^{2}$.

It is the relation derived by Miyazawa. It is again resurrected in our calculation through the assumption that the $U(6 / 21)$ symmetry is broken by an operator that behaves itself like the $s=0, I=0$ member of the $35 \times 35$ representation of $S U(6)$, which was true to $5 \%$. This corresponds to the confined quark approximation with $\alpha_{s}=0$.

In addition, our potential model gives a more accurate symmetry breaking $\left(\alpha_{s} \sim 0.2\right)$ :

$\frac{9}{8}\left(m_{\rho}^{2}-m_{\pi}^{2}\right)=m_{\Delta}^{2}-m_{N}^{2}$

with an accuracy of $1 \%$.

This mass-squared formula arises from the secondorder iteration of the $q-D, q-\bar{q}$ Dirac equation. The factor $\frac{9}{8}$ comes from

$\frac{1}{2}\left(\frac{4}{3} \alpha_{s}\right)^{2}=\frac{8}{9} \alpha_{s}^{2}$

At this point, it is more instructive to derive a firstorder mass formula. Since the constituent quark mass $M_{q}$ is given by Eq. $(18)(\ell=0)$, we have

$M_{q}=\frac{\mu}{\sqrt{2}}$

so that

$\bar{m}=2 M_{q}\left(1-\frac{\sqrt{2}}{3} \alpha_{s}\right) \simeq 1.8 M_{q}$.

When the baryon is regarded as a $q-D$ system, each constituent gains an effective mass $\frac{1}{2} \mu \rho_{0}$ which was approximately the effective mass of the quark in a meson. Hence, the effective masses of $q$ and $D$ in a baryon are

$m_{q}^{\prime} \simeq M_{q}, \quad m_{D}^{\prime}=M_{D}+M_{q} \simeq 3 M_{q}$.

The spin splittings for a nucleon $N$ and the $\Delta$ are given by the Breit term

$\Delta M=K \Delta V \frac{\boldsymbol{S}_{q} \boldsymbol{S}_{D}}{m_{q}^{\prime} m_{D}^{\prime}}$

For a nucleon with spin $\frac{1}{2}$, the term $\boldsymbol{S}_{q} \cdot \boldsymbol{S}_{D}$ gives -1 , while it has the value $\frac{1}{2}$ for $\Delta$ with spin $\frac{3}{2}$. Using the same $K$ for mesons and baryons which are both considered to be a bound state of a color triplet with a color antitriplet, we can relate the baryon splitting $\Delta M$ to the meson splitting $\Delta m$, for which $\boldsymbol{S}_{q} \cdot \boldsymbol{S}_{\bar{q}}$ takes the values $\frac{1}{4}$ and $\frac{-3}{4}$. Hence, we find

$\Delta M=M_{\Delta}-M_{N}=\frac{3}{2} \frac{K \Delta V}{m_{q}^{\prime} m_{D}^{\prime}}=\frac{1}{2} \frac{K \Delta V}{M_{q}^{2}}$

ISSN 2071-0194. Ukr. J. Phys. 2019. Vol. 64, No. 12 
and

$\Delta m=\frac{K \Delta V}{M_{q}^{2}}$

which leads to a linear mass formula

$\Delta M=\frac{1}{2} \Delta m$,

which is well satisfied and was verified before using the three-quark constituents for a baryon.

The formation of diquarks which behave like antiquarks, as far as QCD is concerned, is crucial to the hadronic supersymmetry and to the quark dynamics for excited hadrons. The splittings in the mass spectrum are well understood on the basis of spindependent terms derived from QCD. This approach to hadronic physics has led to many profound investigations recently. To see the symmetry breaking effect, we note that the mass of a hadron will take the approximate form

$m_{12}=m_{1}+m_{2}+K \frac{\boldsymbol{S}_{1} \boldsymbol{S}_{2}}{m_{1} m_{2}}$,

where $m_{i}$ and $\boldsymbol{S}_{i}(i=1,2)$ are, respectively, the constituent mass and the spin of a quark or a diquark. The spin-dependent Breit term will split the masses of hadrons of different spin values. If we assume $m_{q}=m_{\bar{q}}=m$, where $m$ is the constituent mass of $u$ or $d$ quark, and denote the mass of a diquark by $m_{D}$, then this approximation gives

$$
\begin{aligned}
& m_{\pi}=\left(m_{q \bar{q}}\right)_{s=0}=2 m-K \frac{3}{4 m^{2}}, \\
& m_{\rho}=\left(m_{q \bar{q}}\right)_{s=1}=2 m+K \frac{1}{4 m^{2}}, \\
& m_{\Delta}=\left(m_{q D}\right)_{s=3 / 2}=m+m_{D}+K \frac{1}{2 m m_{D}}, \\
& m_{N}=\left(m_{q D}\right)_{s=1 / 2}=m+m_{D}-K \frac{1}{m m_{D}} .
\end{aligned}
$$

Eliminating $m, m_{D}$, and $K$, we obtain the mass relation

$\frac{8}{3} \frac{2 m_{\Delta}+m_{N}}{3 m_{\rho}+m_{\pi}}=1+\frac{3}{2} \frac{m_{\rho}-m_{\pi}}{m_{\Delta}-m_{N}}$

which agrees with experiment to $8 \%$.

ISSN 2071-0194. Ukr. J. Phys. 2019. Vol. 64, No. 12

\section{Open Questions}

A derivation of an effective relativistic QCD potential from the lattice gauge theory or from analytic techniques is needed. This must await a better understanding of fermion loop corrections and the incorporation of a chiral symmetry. The logarithmic variation of the running coupling constant on $q-\bar{q}$ and $q-D$ spectra has already been considered in the context of a semirelativistic theory. This does not change the qualitative picture presented here. The next step should be the setting up of an effective relativistic theory based on the Dirac equation for the quark and the Klein-Gordon equation for the antiquark, exhibiting the invariance under the relativistic supersymmetry. This might be achieved with the Wess-Zumino Lagrangian constructed out of a gluon field interacting with the vector superfield multiplet (it contains the quark and the antidiquark). The preliminary work in this direction has already been presented in our papers, and the derivations of new mass formulae have shown good agreement with experiments. It is important to attempt calculations of decay widths, magnetic moments, charge radii, and form factors.

The two-body relativistic Hamiltonian with the $S U(6 / 21)$ symmetry between quarks and antidiquarks derived previously can be extended to include the symmetries of three-quark systems. The new Hamiltonian is invariant under the discrete group $S_{3}$. Hence, its eigenstates are eigenstates of $S_{3}$. The splitting of three quarks into $q$ and $D$ will break the $S_{3}$ symmetry down to $Z_{2} \sim S_{2}$ which has two eigenstates. Such considerations should lead to phenomenologically viable models from which hadronic properties can be extracted.

A possible Skyrme model that can compete with the QCD-inspired potential model presented here is still hidden in the future, For a preliminary work in that area, we refer the reader to our paper [32].

It is a pleasure to acknowledge the helpful conversations with Professors Vladimir Akulov, Cestmir Burdik, and Francesco Iachello. One of us (SC) would like to thank Borys Grinyuk for the invitation to the BGL-2019 conference in Kiev and for his kind hospitality.

The present work was supported in part by DOE contracts no. DE-AC-0276-ER 03074 and 03075; NSF Grant No. DMS-8917r54; and several PSCCUNY research grants. 
1. F. Gürsey, L.A. Radicati. Spin and unitary spin independence of strong interactions. Phys. Rev. Lett. 13, 299 (1964).

2. H. Miyazawa. Baryon number changing currents. Prog. Theo. Phys. 36, 1266 (1966).

3. H. Miyazawa. Spinor currents and symmetries of baryons and mesons. Phys. Rev. 170, 1586 (1968).

4. P. Ramond. Dual theory for free fermions. Phys. Rev. D 3 , 2415 (1971).

5. A. Neveu, J. Schwarz. Factorizable dual model of pions science direct. Nucl. Phys. B 31, 86 (1971).

6. F. Gliozzi, J. Scherk, D. Olive. Supersymmetry, supergravity theories and the dual spinor model. Nucl. Phys. B 122, 253 (1977).

7. L. Brink, J. Schwarz, J. Scherk. Supersymmetric YangMills theories. Nucl. Phys. B 121, 77 (1977).

8. T.A. Golfand, E.P. Likthman. Extension of the algebra of Poincare group generators and violation of $\mathrm{p}$ invariance. JETP Lett. 13, 323 (1971).

9. D.V. Volkov, P. Akulov. Is the neutrino a Goldstone particle. Phys. Lett. B 46, 109 (1973).

10. J. Wess, B. Zumino. Supergauge transformations in four dimensions. Nucl. Phys. B 70, 39 (1974).

11. J. Wess, B. Zumino. A Lagrangian model invariant under supergauge transformations. Phys. Lett. B 49, 52 (1974).

12. A. deRujula, H. Georgi, S. Glashow. Hadron masses in a gauge theory. Phys. Rev. D 12, 147 (1975).

13. T. Eguchi. Baryons, diquarks and strings. Phys. Lett. 59, 457 (1975).

14. S. Catto, F. Gürsey. Algebraic treatment of effective supersymmetry. Nuovo Cimento A 86, 201 (1985).

15. S. Catto, F. Gürsey. New realizations of hadronic supersymmetry. Nuovo Cimento A 99, 685 (1988).

16. K. Johnson, C.B. Thorn. Stringlike solutions of the bag model. Phys. Rev. 13, 1934 (1976).

17. P. Hasenfratz, J. Kuti. The quark bag model. Phys. Rep. C 75, 1 (1978).

18. G.F. de Teramond, H.G. Dosh, S.J. Brodsky. Baryon spectrum from superconformal quantum mechanics and its light-front holographic embedding. Phys. Rev. D 91, 045040 (2015).

19. G.F. de Teramond, H.G. Dosh, S.J. Brodsky. Superconformal baryon-meson symmetry and light-front holographic QCD. Phys. Rev. D 91, 085016 (2015).

20. G.F. de Teramond, H.G. Dosh, S.J. Brodsky. Supersymmetry across the light and heavy-light hadronic spectrum. Phys. Rev. D 92, 074010 (2015).
21. G.F. de Teramond, H.G. Dosh, S.J. Brodsky. Light-front holographic QCD and emerging confinement. Phys. Rep. 584, 1 (2015).

22. G.F. de Teramond, H.G. Dosh, S.J. Brodsky. Universal effective hadron dynamics from superconformal algebra. Phys. Lett. B 759, 171 (2016).

23. G.F. de Teramond, H.G. Dosh, S.J. Brodsky. Supersymmetry across the light and heavy-light hadronic spectrum. II. Phys. Rev. D 95, 034016 (2017).

24. S.J. Brodsky, M. Nielsen. Hadronic superpartners from a superconformal and supersymmetric algebra. Phys. Rev. D 97, 114001 (2018).

25. S. Catto. Supergroups in critical dimensions and division algebras. J. Phys. 804, 19 (2017).

26. S. Catto. Role of octonions in hadronic physics. Phys. Part. Nuclei, Lett. 14(2), 6 (2017).

27. S. Catto. Quantum symmetries: From Clifford and Hurwitz algebras to M-theory and leech lattices. Advances in Applied Clifford Algebras 28, 81 (2018).

28. M. Anselmino et al. Diquarks. Rev. Mod. Phys. 65, 1199 (1993).

29. F. Iachello. Algebraic models of hadronic structure. Nucl. Phys. A 497, 23 (1989).

30. F. Iachello, N. Mukhopadhyay, L. Zhang. Spectrumgenerating algebra for stringlike mesons: Mass formula for $q \bar{q}$ mesons. Phys. Rev. B 44, 898 (1991).

31. F. Iachello. Anales de Fisica. Monographias 1, 13 (1993).

32. S. Catto, H.Y. Cheung, F. Gürsey. Effective hamiltonian of the relativistic quark model. Mod. Phys. Lett. A 6, 3485 (1991).

Received 02.09.19

С. Катто, І. Гюрчан, Б. Ніколеску, Е. Ю

ЕФЕКТИВНА ГАДРОННА

СУПЕРСИМЕТРІЯ 3 КВАНТОВӦ̈

ХРОМОДИНАМІКИ

$\mathrm{P}$ е $з$ ю м е

Кваркова модель з потенціалами, отриманими з КХД, яка включає модель антикварк-дікварка для збуджених адронів, дає масові формули, що дуже добре узгоджуються 3 експериментом. Використовуються наближені симетрії та суперсиметрії гадронного спектру включно із механізмом порушення симетрії. 